\section{Antibiotic resistance}

\section{Antimicrobial stewardship and the anxious patient}

Sir, the current issue of antimicrobial stewardship is an important and necessary aspect of healthcare in which we as dentists have a crucial role to play.

The BDA, FGDP and Public Health

England have launched action plans to tackle this issue of antibiotic resistance, and require healthcare professionals to be engaged. ${ }^{1}$

Without urgent action, there is a real threat of entering a post antibiotic era as antibiotics are becoming less effective. ${ }^{2}$

As guided by NICE, we must avoid overuse and misuse of antibiotics. ${ }^{3}$ Ensuring appropriate use of antibiotics is crucial to preventing resistance and protecting patients, as well as cutting down unnecessary costs. We must continue to educate our patients about resistance, and why prescribing may not be the ideal treatment modality compared to local intervention.

Standard 1.4 as outlined by the GDC, advises us to provide patients with treatment that is in their best interests, following clinical guidelines relevant to their situation. We are advised to balance their oral health needs with their desired outcomes.

A recurrent difficulty faced by many practitioners is management of anxious patients. These patients simply cannot tolerate any conventional interventions, such as incision and drainage of a swelling, access through the tooth, or an extraction.

This cohort of patients presents practitioners with what at times appears an insoluble problem - where the patient demands are in conflict with ideal local treatment. This leaves us with an ethical dilemma - although patients have autonomy to deny treatment, to leave a patient in pain or with a swelling is both unethical and unacceptable. This could lead to deterioration in health with potentially serious consequences.

Recent media attention, and campaigns developed by the FGDP have been invaluable in educating patients on how 'antibiotics don't cure toothache?.

However, whilst time invested in education of the patient and advice regarding their role in antibiotic stewardship is important, the present contracting arrangements within primary care make this challenging.

With time limitations, stressful UDA targets and ever-increasing patient demands, dentists may feel backed into a corner. One might question what alternatives exist to manage an anxious patient that presents in a time-limited pain slot.

\section{K. Smorthit and T. Thayer, Liverpool, UK,} by email

\section{References}

1. BDA. Antibiotic resistance in dentistry. 2018. Available at https://bda.org/amr (accessed April 2019).

2. World Health Organization. Antibiotic Resistance. 2018 Available at https://www.who.int/news-room/factsheets/detail/antibiotic-resistance (accessed April 2019).

3. NICE guidance (NG15) 2018. Antimicrobial stewardship: systems and processes for effective antimicrobial medicine use. Available at https://www.nice.org.uk/ guidance/ng15 (accessed April 2019).

DOI: $10.1038 / \mathrm{s} 41415-019-0322-7$

\section{Stress}

\section{Emotional burnout}

Sir, burnout scores for emotional exhaustion in UK doctors ${ }^{1}$ and the recent paper by Toon et al. ${ }^{2}$ indicate that many dentists suffer bad stress due to organisational and personal factors. It is surely urgent that research focuses on how stress pathways develop throughout medical careers.

Taking note of studies in dental education which look at the development of professionalism could well be a good way forward.

Strong reactive emotions in dental students such as fear, frustration and shame have been found to be associated with challenging patient encounters and taking more responsibility for care. ${ }^{3}$ In my experience, many foundation dentist peers find these sorts of strong emotions can really hinder us, yet are often hard to admit for fear of being seen as weak.

The culture in medicine is hopefully now changing towards accepting that emotional sensitivity is part of being a good dentist. Current anti-stigma campaigns also attempt to reduce barriers to admitting stress or illness in medics and denitsts. ${ }^{4}$

If research looks into our emotional reactions to provide more of an understanding of dentists' stress drivers, and if the medical culture can adapt, burnout rates may start to fall.

R. Bernstein, Budleigh Salterton, UK, by email

\section{References}

1. Imo U O. Burnout and psychiatric morbidity among doctors in the UK: a systematic literature review of prevalence and associated factors. BJ Psych Bulletin 2017; 41: 197-204.
2. Toon M, Collin V, Whitehead P, Reynolds L. An analysis of stress and burnout in UK general dental practitioners: subdimensions and causes. Br Dent J 2019; 226: 125-130.

3. Ranauta A, Freeth D, Davenport E. Developing understanding and enactment of professionalism: undergraduate dental students' perceptions of influential experiences in this process. Br Dent Journal 2018; 225: 662-666.

4. Doctor's Support Network. \&me Anti Stigma Campaign. 2019. Available at https://www.dsn.org.uk/ANDME_ anti_stigma_campaign (accessed April 2019).

DOI: $10.1038 / 541415-019-0321-8$

\section{LGBT+ recognition}

\section{Why I refuse the rainbow lanyard}

Sir, I have been asked to consider wearing a rainbow lanyard at work 'to send a clear message of support to the LGBT+ community.' ${ }^{1}$

My gut reaction was to agree, to help increase visibility for a community I support. I firmly believe in equalities in health and so on first glance this appears like a very worthy idea, especially when considering that more than $20 \%$ of LGBT+ people feel uncomfortable being open about their sexual orientation or gender identity with NHS staff. ${ }^{2}$

There was, however, an underlying discomfort in me about the request that I feel warrants discussion. I became concerned about the potential distress to other patient groups caused by such a personal display of support for the LGBT+ community, with an item worn in the clinical setting.

When I referred to the GDC Standards ${ }^{3}$ I found that Standard 1.6.4 states: 'You must not express your personal beliefs (including political, religious or moral beliefs) to patients in any way that could cause them distress.'

Whilst $2 \%$ of the UK population identifies as lesbian, gay or bisexual, ${ }^{4}$ the last census found $59 \%$ of the population identifying as Christian. ${ }^{5}$

The dominant Christian churches in the UK are clear on their stance towards homosexuality. The Church of England the state religion of England - maintains that homosexuality is 'incompatible with Scripture, ${ }^{6}$ and in a 2017 interview the Archbishop of Canterbury, Justin Welby was unable to answer whether gay sex is sinful. ${ }^{7}$

The Roman Catholic Church asserts that homosexuality is a 'strong tendency ordered toward an intrinsic moral evil; and thus the inclination itself must be seen as an objective disorder. ${ }^{8}$

It should come as little surprise then to find that $25 \%$ of Christians disagree with same-sex marriage, ${ }^{9}$ and that $12 \%$ of the 